\title{
Diagnosis of scaphoid fracture: optimal imaging techniques
}

\author{
This article was published in the following Dove Press journal: \\ Reports in Medical Imaging \\ 30 July 2013 \\ Number of times this article has been viewed
}

\section{Mats Geijer}

Center for Medical Imaging and Physiology, Skåne University Hospital and Lund University, Lund, Sweden
Correspondence: Mats Geijer

Center for Medical Imaging and Physiology, Skåne University Hospital, S-22I 85 Lund, Sweden

Tel +46 $46 \quad 173083$

Fax +46 46176036

Email mats@geijer.info
Abstract: This review aims to provide an overview of modern imaging techniques for evaluation of scaphoid fracture, with emphasis on occult fractures and an outlook on the possible evolution of imaging; it also gives an overview of the pathologic and anatomic basis for selection of techniques. Displaced scaphoid fractures detected by wrist radiography, with or without special scaphoid views, pose no diagnostic problems. After wrist trauma with clinically suspected scaphoid fracture and normal scaphoid radiography, most patients will have no clinically important fracture. Between $5 \%$ and $19 \%$ of patients (on average $16 \%$ in meta-analyses) will, however, have an occult scaphoid fracture which, untreated, may lead to later, potentially devastating, complications. Follow-up imaging may be done with repeat radiography, tomosynthesis, computed tomography, magnetic resonance imaging (MRI), or bone scintigraphy. However, no method is perfect, and choice of imaging may be based on availability, cost, perceived accuracy, or personal preference. Generally, MRI and bone scintigraphy are regarded as the most sensitive modalities, but both are flawed by false positive results at various rates.

Keywords: occult fracture, wrist, radiography, computed tomography, magnetic resonance imaging, radionuclide imaging

\section{Introduction}

The small carpal scaphoid presents a challenge both for imaging and for treatment after trauma. Traditionally, the wrist is imaged with posterior-anterior (PA) and lateral radiographic views after trauma such as a fall on an outstretched hand. When a scaphoid fracture is suspected, supplementary scaphoid views are usually performed. Radiography with scaphoid views is not perfect, however, since it fails to detect all fractures, and the sensitivity is low to moderate. In some patients with clinical symptoms of scaphoid fracture and negative radiography, occult fractures may remain. Such patients should be followed up with to avoid complications later.

The purpose of the current paper is to give an overview of imaging modalities used to image the scaphoid bone and to discuss the possible strategies in following up patients suspected of having an occult scaphoid fracture - a fracture not detectable with the imaging modality used, in this instance, radiography.

\section{Anatomy}

The carpal scaphoid is one of eight carpal bones, articulating with the radius, lunate, capitate, trapezium, and trapezoid. It is the largest carpal bone, with a mean long axis length of $27.1 \mathrm{~mm}$ in males, and $23.9 \mathrm{~mm}$ in women. ${ }^{1}$ With the hand extended, the slightly bowed scaphoid bone sits at about a 45-degree angle in both the anterior 
posterior and lateral planes. With radial deviation of the hand, it rotates palmarly to give room for the trapezium and trapezoid, and with ulnar deviation it rotates dorsally. Anatomically, the scaphoid forms a bridge between the proximal and distal carpal row and provides mechanical stability.

About $80 \%$ of the surface of the scaphoid bone is joint surface covered with hyaline cartilage, which has implications for imaging, post-traumatic complications, and surgical treatment. This means that healing is almost entirely endosteal, and there will be practically no periosteal callus formation due to the cartilage-covered bony surface. Thus, healing is slower than in bones that also have periosteal callus formation while at the same time being harder to evaluate since only the endosteal callus formation and eventually the bridging trabecular bone formation are reliable indicators of healing. The blood supply comes mainly from a single branch of the radial artery, which enters distally and usually by dividing into two smaller branches which traverse the dorsal cortex distal to the waist of the scaphoid. The almost entirely intraosseous vascular blood supply to the proximal half of the scaphoid carries with it the risk that a displaced waist fracture will sever arteries and veins supplying the proximal pole fragment, which will result in avascular necrosis..$^{2-7}$ The smaller the proximal pole fragment, the higher the risk is considered to be for proximal pole avascular necrosis. Surgical treatment has advanced in recent years and is focused on restoring correct anatomy and good contact between the fracture fragments to prevent nonunion and avascular necrosis.

\section{Trauma mechanisms and treatment}

The fracture mechanisms for scaphoid fractures are well known. The fracture usually results from a fall on an outstretched arm with the hand in dorsal flexion, with the dorsal lip of the distal radius acting as a fulcrum. It has been suggested that the mechanism may be a backward fall on a dorsiflexed wrist in ulnar deviation. ${ }^{8}$ Other mechanisms have been described, such as a soccer goalkeeper receiving a ball to the hand or after other types of axial trauma to the hand. Scaphoid fractures may be classified on their orientation as horizontal oblique, transverse, and vertical oblique (Russe classification). ${ }^{9}$ Another system classifies them as acute stable, acute unstable, delayed union, and non-union (Herbert classification). ${ }^{10-12}$ There are several other fracture classifications of varying complexity. ${ }^{13}$ For a full understanding of the multiple fracture types, a three-dimensional (3D) understanding is necessary. ${ }^{13}$ To avoid poor outcome after scaphoid fractures, proper and timely imaging is vital, ${ }^{14}$ as late fracture detection and failure to recognize displacement are associated with increased non-union. ${ }^{15,16}$ Almost $70 \%$ of scaphoid fractures have been reported as unstable. ${ }^{17}$

Relatively insignificant in clinical terms, avulsions of the distal tuberosity carry no risk of significant non-union or avascular necrosis, usually heal without complications, and are not the subject of this review. There have been, however, cases reported with poor healing. ${ }^{18}$

Most non-displaced scaphoid waist fractures heal without complications after treatment with a cast for about 6 weeks ${ }^{19,20}$ to 12 weeks, ${ }^{14,21,22}$ and rates of up to $95 \%$ healing for cast treated scaphoid waist fractures have been reported ${ }^{23}$ Fractures with a displacement of more than 1-2 mm increasingly receive internal fixation with pins or compression screws ${ }^{24}$ to avoid complications such as avascular necrosis or nonunion, ${ }^{25}$ as do patients with bilateral fractures. Cases with a displacement of more than $1 \mathrm{~mm}$ are associated with complications such as non-union (55\%) and avascular necrosis $(50 \%) .{ }^{25}$ Proximal pole fractures are prone to complications, where surgical treatment is often advocated. ${ }^{26}$ The question of whether patients with high functional demands should have stable and non-displaced fractures fixated is still under debate, as well as whether internal fixation - despite higher direct costs - is beneficial from a societal economic perspective. ${ }^{14}$ Internal fixation also leads to more complications, even if they are minor. ${ }^{27} \mathrm{~A}$ meta-analysis suggests that surgical treatment of non-displaced or minimally-displaced fractures improves functional outcome and reduces time off from work, at the expense of increased complications. ${ }^{27}$ Fractures with non-union have the potential of progression into a scaphoid non-union advanced collapse (SNAC) wrist, ${ }^{7,28}$ which is a devastating condition. To prevent this, internal fixation with bone graft is performed, with about an $85 \%$ success rate if performed in a timely fashion.

Due to the potential for severe complications arising from a missed occult scaphoid fracture, all patients with clinical signs of scaphoid fracture are usually treated with a cast until at least follow-up imaging has been done, even if no fracture is eventually diagnosed with imaging. This means that about $80 \%$ of these patients are treated unnecessarily. ${ }^{29-31}$ The ideal role for imaging is to provide a solid basis for treatment decision, with all fractures detected promptly and without over-diagnosis leading to unnecessary treatments.

\section{Population statistics}

Waist fractures of the scaphoid occur most often in young male adults engaged in sporting activities or otherwise subjected to high energy trauma. Waist fractures are rare in children due to the greater plasticity of the growing skeleton. 
However, even though rare, waist fractures in children may also result in non-union. ${ }^{32}$

The scaphoid is the most frequently injured carpal bone. ${ }^{33-37}$ In a study from Bergen in Norway, scaphoid fractures accounted for between $2 \%$ and $3 \%$ of all fractures, about $11 \%$ of wrist fractures, and about $60 \%$ of all carpal fractures. ${ }^{33}$ The age-specific incidence of scaphoid fractures peaks in late adolescence and early adult life. ${ }^{33}$ In Norway it was reported that the peak for females is in the 10-19-year-old age group, with an annual incidence of 4.6 per 10,000 female subjects; for males the peak was in the 20-29-year-old age group, with an annual incidence of 15.1 per 10,000 subjects. ${ }^{33}$ The average annual incidence for the entire population was 1.5 per 10,000 females and 7.3 per 10,000 males. In a study from Denmark, the reported average annual incidence was 0.8 per 10,000 females and 3.8 per 10,000 males. Among the younger children between 10 and 14 years, the average annual incidence was 0.3 per 10,000 girls and 3.9 per 10,000 boys. ${ }^{38}$ Concomitant distal radius and scaphoid fractures are rare, consisting of about $3.1 \%$ of all scaphoid fractures, ${ }^{39-45}$ they are seen mostly in patients with high-energy trauma or severe osteoporosis.

\section{Clinical diagnosis}

The clinical presentation of a scaphoid fracture is variable, but in nearly all cases there will be wrist pain and tenderness. Several clinical signs have been described, ${ }^{46-48}$ such as snuff box tenderness, tenderness over the scaphoid tubercle, ${ }^{49-51}$ or tenderness with axial compression of the first or second digits (the scaphoid compression test). ${ }^{52}$ Combining the clinical tests improves the clinical diagnosis, which has a high sensitivity. However, the specificity is only between $74 \%$ and $80 \% .^{53,54}$

\section{Modern imaging and reference standards}

There is currently no consensus on which reference standard is the optimal to use in scientific research on scaphoid fracture imaging. ${ }^{55,56}$ In many studies 6-week follow-up imaging with radiography is used; such an approach has been viewed as a poor reference standard ${ }^{57}$ since potential later displacements may be missed. Latent class analysis has been suggested as a way to circumvent this problem. ${ }^{55,56}$ In many studies in the clinical setting, reference imaging with a gold standard may be impractical if the study population is too large; conversely, complete reference imaging may not be available in a retrospective study. A longer follow-up time, such as 1-year follow-up, may be used, provided that all patients can be followed up with in the medical and/or imaging records, ${ }^{58}$ and presuming that all clinically significant complications will have presented by then. Magnetic resonance imaging (MRI) is commonly used as reference in prospective studies on smaller clinical materials, but true discrimination between a cortical fracture with potential instability and a simple trabecular fracture with intact cortex may be impossible. In cases with intact joint cartilage, even arthroscopy cannot be relied upon completely for fracture diagnosis.

\section{Radiography}

Conventional radiography of the wrist with special scaphoid views has been and remains the best way to initially evaluate a patient suspected of having a scaphoid fracture.

Optimal imaging requires well-exposed PA and lateral views of the wrist in neutral rotation; ${ }^{59,60}$ it is also important to avoid radial deviation on the PA view, as this makes adequate imaging of the scaphoid impossible. The wrist images should be supplemented by 2-4 special views of the scaphoid, where more than two dozen different projections have been described (Figure 1). ${ }^{61}$ The fact that a mid-waist scaphoid fracture is oriented at about 45 degrees to the orthogonal planes combined with the fact that a fracture line as narrow as 1-2 mm constitutes a displaced fracture with high potential for non-union makes it is easy to understand why there are such difficulties in detecting these clinically important fractures using radiography. A non-displaced or minimally displaced fracture can only be detected when the X-ray beam is parallel to the fracture line (Figure 1). If the X-ray beam is only a few degrees oblique to the fracture, it may very well remain undetected. It is not uncommon that a scaphoid fracture is seen on only one of four scaphoid views.

Radiography is cheap, readily available at all hours, and the radiation dose is negligible. Most scaphoid fractures

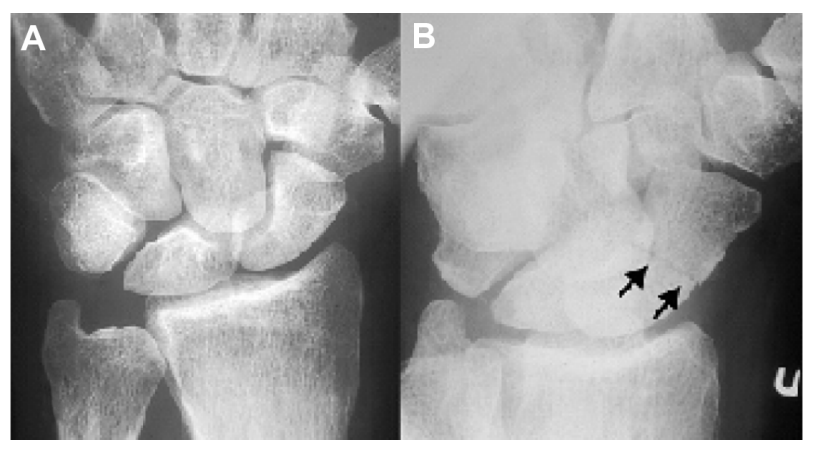

Figure I A 5 I-year-old male with trauma to the wrist.

Notes: (A) The posterior-anterior wrist radiograph was unremarkable. (B) The minimally displaced hairline waist fracture was displayed only on one scaphoid view (arrows). The patient was subsequently operated on, with pinning, with good results. 
will be detected, making further imaging unnecessary for diagnosis. Computed tomography (CT) scans may be added if improved fracture delineation is important for surgical planning. Besides scaphoid fractures, nearly all distal radius fractures will be detected, as well as a number of other carpal and metacarpal fractures. There are limitations with using radiography in young patients where there is incomplete ossification of the scaphoid cartilage precursor, as well as in old osteoporotic patients, where there may be issues with limited image quality.

Radiography with scaphoid views is not perfect, however, and the sensitivity is low to moderate. It has been reported as $35 \%$ in one study with bone scintigraphy as reference, ${ }^{62}$ with higher figures in other studies, whereas the specificity in contrast is high. The prevalence of occult scaphoid fractures after trauma thus varies in different reports between $5 \%$ and $19 \%$, and on average $16 \%$ in meta-analyses. ${ }^{63-65}$ Occult fractures may persist even at the 2-week follow-up examiniation, ${ }^{58,61}$ and in a meta-analysis repeat radiography was reported as having a sensitivity of $91.1 \%$ and specificity of $99.8 \% .{ }^{56}$ However, treating all patients with clinical symptoms and negative radiography as actual fracture cases would result in unnecessary immobilization in casts and a substantial loss of productivity and income in about $84 \%$ of these patients. ${ }^{31,66}$

The prevalence of occult fractures in patients with clinical symptoms of scaphoid fracture and normal radiography will vary between studies depending on the type, quality, and accuracy of the clinical examination and the selected cutoff level for severity of symptoms to be called fracture-suspect. The prevalence will also vary depending on the radiography performed (general image quality, spatial resolution, number and types of scaphoid views, the observers' level of expertise, and selected cutoff level for calling a fracture) and the actual prevalence of fractures, since this has an impact on the number of false-positive and false-negative findings.

\section{Tomosynthesis}

Linear digital tomosynthesis ${ }^{67}$ is a modern digital version of conventional tomography wherein radiography is augmented by tomographic images. At present, three vendors supply digital tomosynthesis for whole-body imaging, whereas multiple vendors provide tomosynthesis for mammography. ${ }^{68}$

In digital tomosynthesis from one vendor, tomographic images are generated from multiple low-dose exposures directed towards a stationary digital amorphous silicon flatpanel detector, from tube angles of $-15^{\circ}$ to $+15^{\circ}$ of a moving $\mathrm{X}$-ray tube. The tomosynthesis function is provided as a software add-on to a conventional, fully-motorized radiography system. The exposures are used to reconstruct up to 60 coronal sectional images with a nominal thickness between 1 and $10 \mathrm{~mm}$. The structures included in each sectional image are sharply depicted, while structures located anteriorly or posteriorly to the section are blurred. The tomographic fracture diagnosis is mainly based on cortical disruption (Figure 2) since anatomic noise disturbs the viewing of the trabecular bone. However, endosteal callus formation in non-displaced fractures may be seen (Figure 3).

Tomosynthesis is performed during the same visit and in the same room as radiography, and is thus a very simple and time-efficient way to improve on radiography. The added cost of performing tomosynthesis is low, and the concern for added radiation is low when investigating a peripheral object such as the wrist in adults. Tomosynthesis thus appears to be a quick and easy way to further strengthen the radiographic diagnosis and help in deciding on further investigations and treatment.
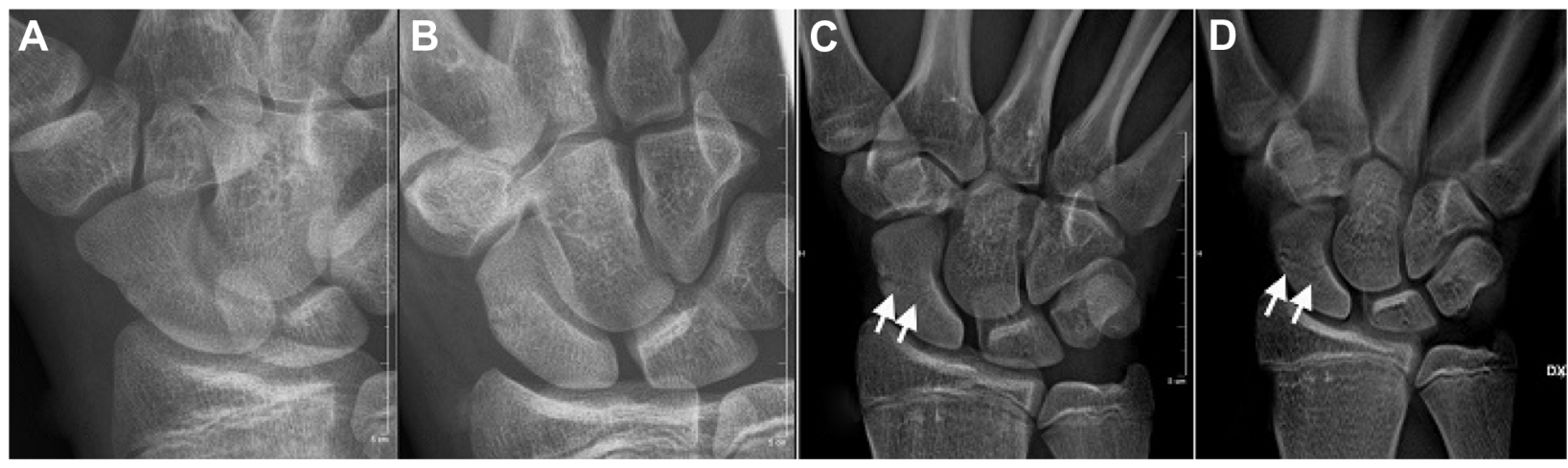

Figure 2 A 15-year-old boy with wrist trauma.

Notes: (A and B) Initially unremarkable radiographs. (C) A non-displaced fracture was visualized (arrows) at follow-up after 2 weeks with radiography. (D) The fracture was better delineated with tomosynthesis (arrows). 


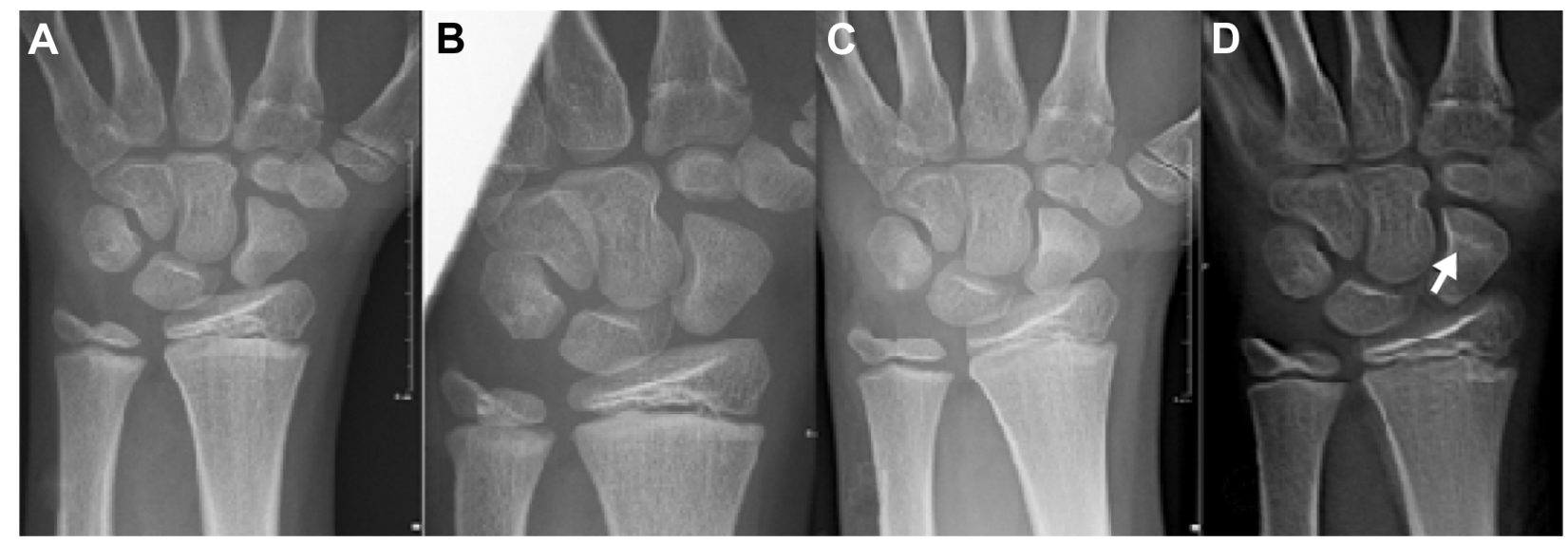

Figure 3 An I I-year-old girl with wrist trauma.

Notes: (A and B) Initially unremarkable radiographs, including the scaphoid views. (C) After 2 weeks, radiography was still unremarkable. (D) Tomosynthesis displayed a sclerotic band across the scaphoid waist, consistent with callus formation in an incomplete scaphoid fracture (arrow).

In a study on 35 patients returning for a 2-week's followup visit with initially normal radiography but suspected of having an occult scaphoid fracture, ${ }^{58}$ repeat radiography revealed one previously undetected scaphoid tubercle avulsion and one scaphoid waist fracture, both confirmed by tomosynthesis. The tomosynthesis scans revealed two additional patients with scaphoid waist fractures. In total, three initially occult scaphoid waist fractures were detected (9\%). Clinical and radiologic follow-up was used as reference, with no additional fractures detected in the remaining 32 patients during a 1-year follow-up. In another study on wrist trauma, 100 of 2,386 patients seen in the emergency department had had supplementary imaging with tomosynthesis and CT. ${ }^{69}$ Fifty-seven of the 100 patients had a fracture, $19 \%$ of the scaphoid bone. Sensitivity for three readers was $61 \%-80 \%$ for radiography, $77 \%-87 \%$ for tomosynthesis, and $93 \%-95 \%$ for CT. Specificity was $65 \%-83 \%, 76 \%-82 \%$, and $86 \%-95 \%$, respectively, with tomosynthesis performing better than radiography but worse than CT.

Tomosynthesis should not be seen as a "poor man's CT" but rather as an extension and augmentation of conventional radiography.

\section{Computed tomography}

CT is an excellent method to follow up with patients suspected of having an occult scaphoid fracture after wrist trauma (Figure 4).

Imaging should be done with as narrow a beam and detector collimation as possible for optimal image quality, enough dose to keep the noise level acceptable, and should be reconstructed with a small field-of-view (FOV) ${ }^{70}$ If $3 \mathrm{D}$ imaging is contemplated, a soft reconstruction kernel should be used. ${ }^{70}$ Any particular patient positioning other than keeping the hand above the head is not important, since the most common fracture extension (a transverse waist fracture) will be more or less oblique to the axial scanning plane, which improves the detectability of fractures on multiplanar reformations (MPR) and follows the principle of obliquity. ${ }^{71}$ MPR reconstructions of 1-2 mm thickness should be performed in the three orthogonal planes for general evaluation of the entire wrist, including the distal radius and ulna, and the scaphoid should be evaluated with special reconstructions along its waist. ${ }^{72-74} \mathrm{As}$ long as the patient can keep the injured wrist above the head the radiation dose to adults is negligible, since the irradiated wrist does not contain radiation-sensitive red bone marrow. The effective radiation dose has been reported as $0.03 \mathrm{mSv} .{ }^{75}$

$\mathrm{CT}$ is available in most institutions around the clock at a moderate cost. CT fracture detection is based mainly on detection of cortical disruptions (Figure 5); the possibility

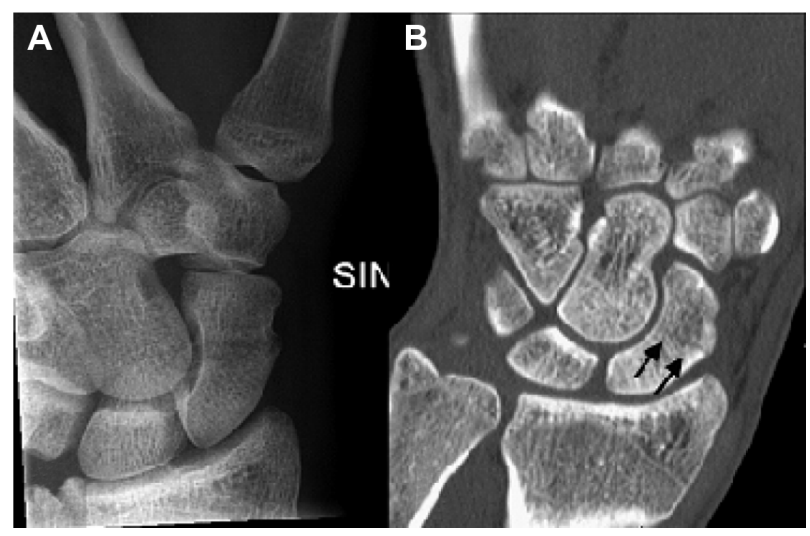

Figure 4 A 26-year-old male with wrist trauma.

Notes: (A) At radiography, one scaphoid view was inconclusive. (B) CT the same day revealed a non-displaced fracture (arrows).

Abbreviation: CT, computed tomography. 


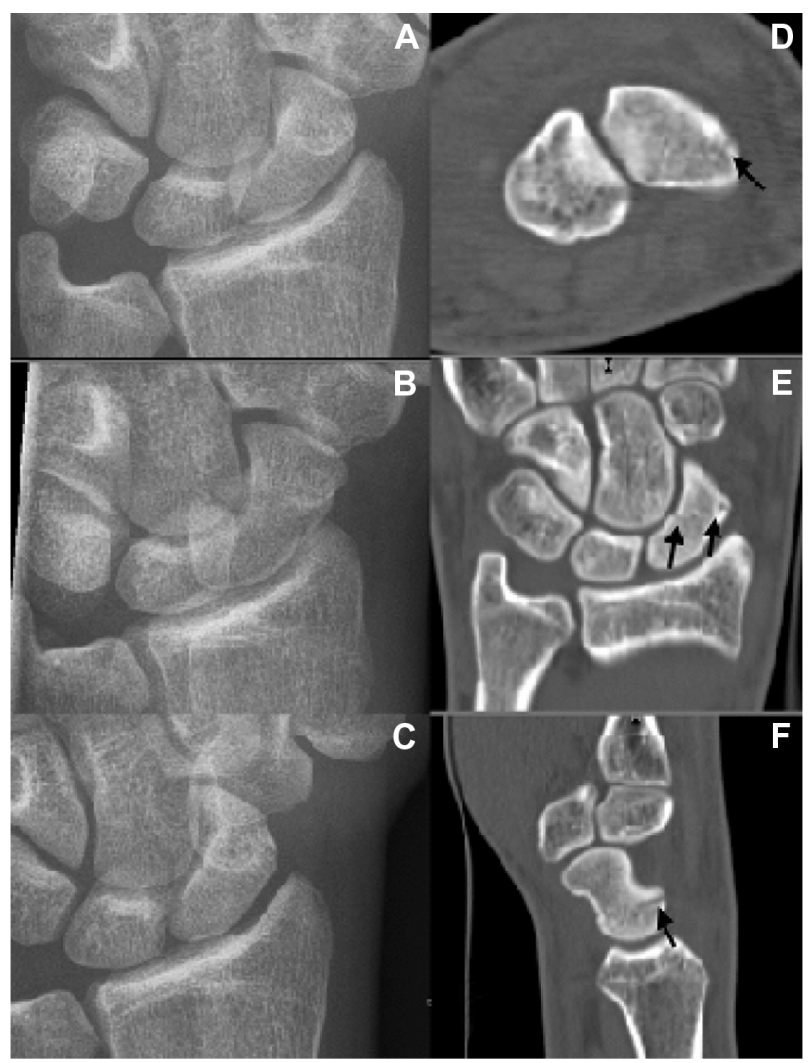

Figure 5 A 20-year-old male with wrist trauma.

Notes: (A, B and C) Radiography was inconclusive, with a suggestion of a hairline fracture. (D, E and F) The fracture suspicion was confirmed by CT the same day, showing a non-displaced fracture (arrows).

Abbreviation: CT, computed tomography.

of using other signs such as bone bruise reported for other types of fracture ${ }^{76}$ is low since the bone is small, there is no side-by-side comparison to the non-injured side, and the patients are usually teenagers or young adults with a fairly dense trabecular bone, meaning the bone bruise does not show as well as in osteoporotic patients. Dual-energy CT could possibly have an advantage as the dense calcium can then be subtracted from the images. ${ }^{77}$

$\mathrm{CT}$ has the advantage over MRI in having a higher spatial resolution and the possibility to reconstruct MPR images in any arbitrary viewing plane. In addition to fracture detection, $\mathrm{CT}$ is useful for evaluation of fracture healing; additionally, with metallic implants, CT is useful for evaluation of pseudarthrosis or non-union prior to reconstructive surgery, and it is also useful for evaluating postoperative complications. A cast made from plaster or plastic is no obstacle to imaging, and using modern scanners with modern metallic artefact reduction techniques removes problems with imaging patients who have metallic implants.

Sub-acute CT after wrist trauma with a suspected scaphoid fracture revealed $36 \%$ abnormal CT scans in a study on 84 patients, with $7 \%$ occult scaphoid fractures. ${ }^{78}$ The authors concluded that early CT was valuable in detecting fractures and reducing unnecessary immobilization. In a comparison between CT and MRI on 29 patients with 6-week radiographs as reference, ${ }^{79}$ radiography revealed eleven fractures. Three of these had only a band-like lucency within the trabecular portion of the scaphoid. MRI detected all eleven fractures with only two scored as definite cortical fractures, while CT detected all eight cortical fractures but missed the three purely trabecular fractures. Statistically, no significant difference was found between CT and MRI for fracture detection, but for detection of cortical fractures. The sensitivity for detecting occult fractures was reported as $73 \%,{ }^{79}$ the difference compared to MRI was not significant, and the specificity was reported as $100 \%$. In another study comparing CT with MRI, 34 patients with normal radiography and suspected of having an occult scaphoid fracture were examined by a panel of three observers who scored the studies in consensus, ${ }^{29}$ with the 6-week radiographs used as reference. A number of both $\mathrm{CT}$ and MRI studies were scored as false negative or false positive, and the study concluded that both CT and MRI were better at ruling out fractures than confirming them; the study also concluded that the modalities had comparable diagnostic characteristics. The sensitivity for scaphoid fractures was $67 \%$ both for $\mathrm{CT}$ and MRI, while the specificity was $96 \%$ and $89 \%$, respectively.

\section{Extremity CT}

Recently, cone-beam CT, which is normally used for dental imaging, has also been applied in dedicated extremity CT scanners. With an extremity scanner the hand, wrist, and elbow in the upper extremity, and the foot, ankle, knee in the lower extremity, may be examined with higher spatial resolution than with conventional CT. ${ }^{80}$ The lower extremity can be evaluated under weight-bearing conditions, since the gantry can tilt 90 degrees. No studies on wrist or trauma imaging have been published, but a study on wrist arthrography ${ }^{81}$ reported good results, comparable to those from MRI, with extremity CT providing isotropic imaging at $0.4 \mathrm{~mm}^{3}$ voxel size. Cone-beam CT using $\mathrm{C}$-arm fluoroscopy units equipped with digital detectors have been used to provide imaging in image-assisted navigation for scaphoid drilling. ${ }^{82,83}$ Such applications are, however, different from diagnostic conebeam CTs, which use dedicated units derived from originally dental cone-beam CT.

\section{Isotope scanning}

Bone scintigraphy has for long been an excellent method to detect skeletal injury such as scaphoid fractures in younger 
non-osteoporotic patients and where there is rapid onset of healing after trauma.

For bone scintigraphy to be able to detect a scaphoid fracture, a reparative process with osteoblast activity is needed. An example of such a process is endosteal callus formation, in which the radioactive istotope is included in the newly formed callus. As a result, imaging cannot be done until 1-3 days after trauma. ${ }^{84,85}$ The synthetic radioactive istotope 99 mTc-methylene diphosphonate binds by chemiadsorption to hydroxiapatite crystals. About $50 \%$ of the injected dose is accumulated in the skeleton within 2-6 hours after intravenous injection, ${ }^{85}$ which means that the examination is time consuming for the patient, who is usually imaged 4 hours after injection. The effective radiation dose is higher than for CT or radiography (about $4 \mathrm{mSv}$ ), which is equal to 2 years' natural background radiation. The anatomic resolution is adequate for scaphoid fracture diagnosis (Figure 6), and the method is also useful for evaluating the entire carpus and wrist for fractures. ${ }^{86}$

Bone scintigraphy has been reported as having 100\% sensitivity and between $92 \%$ and $98 \%$ specificity in detecting scaphoid fractures. ${ }^{86-88}$ In a study comparing bone scintigraphy and MRI, ${ }^{89} 43$ patients were imaged on average 19 days after wrist trauma. Six patients had scaphoid waist fractures with both modalities, and both modalities detected six additional fractures. The bone scan was false positive in three patients, with the positive scan resulting from osteoarthritis in two cases and soft tissue injury in one case. Sensitivity and specificity for MRI was reported as $100 \%$, while bone scintigraphy had a sensitivity of $83 \%$ and a specificity of $95 \%$. In another study comparing bone scintigraphy with CT on 51 patients imaged 10-14 days after trauma, ${ }^{90}$ bone scintigraphy was positive for ten scaphoid fractures and 13 other carpal or wrist fractures, whereas CT detected five scaphoid fractures and nine other fractures.

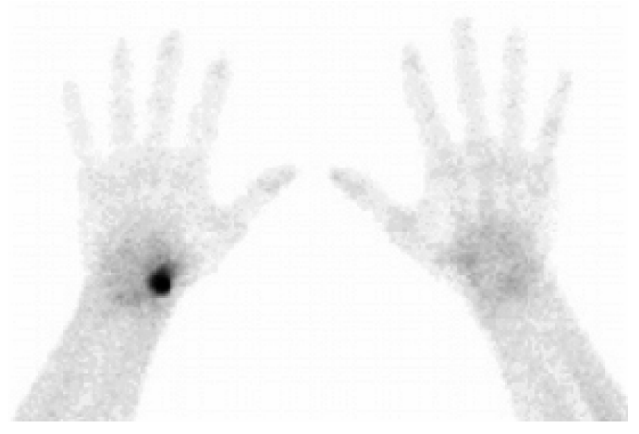

Figure 6 Bone scintigraphy of an 18-year-old man I week after a wrist trauma. Note: Unremarkable radiographs revealed a hot spot in the right scaphoid bone, consistent with an acute scaphoid fracture.
One of the drawbacks of bone scintigraphy is its poor spatial resolution. For example, osteoarthritis in the scaphotrapezotrapezoidal (STT) joint cannot be reliably distinguished from a distal pole scaphoid fracture. Fusion imaging with radiography has been suggested as a way to improve on this weakness, ${ }^{91}$ wherein images similar to other fusion techniques such as positron-emission tomography (PET)/CT or single-photon emission computed tomography (SPECT)/CT are generated, only using radiography as the anatomic basis instead of CT. The adding of fused images to the evaluation of scaphoid bone scintigraphy changed the interpretation of the studies in a significant number of cases, mostly by reducing the number of positive cases and raising interobserver agreement and diagnostic confidence..$^{91}$

\section{Single-photon emission computed tomography/CT}

Hybrid imaging combining physiologic data from bone scintigraphy with anatomic data from CT in the same image is another way to create fusion images. For some years now, SPECT/CT has been available for augmentation of planar bone scanning; images from axial scans with a gamma camera are fused with $\mathrm{CT}$ images to provide new MPR images. ${ }^{85}$ This technique is used mostly for oncologic imaging, but can also be used for evaluation of infection and fractures, utilizing different tracers for different indications. SPECT/CT has the potential to increase the specificity of bone scanning with axial SPECT data fused with CT data presenting both physiologic and anatomic information in one image section.

\section{Ultrasound}

Ultrasound has the potential to differentiate the cause of residual pain between cortical fracture, hemarthrosis, and hematoma. A drawback is that the technique is examinerdependent and time-consuming. The use of ultrasound in suspected scaphoid fracture has been evaluated in one report $^{92}$ which showed a sensitivity of $100 \%$ and specificity of $98 \%$ in 54 patients when using cortical disruption as a diagnostic criterion. In a review, however, ultrasound showed inferior results compared with CT, MRI, or bone scanning. ${ }^{93}$

\section{Magnetic resonance imaging}

MRI (Figure 7) has come to be generally regarded as the gold standard for detection of occult scaphoid fractures, ${ }^{94}$ with a sensitivity and specificity of $100 \%$ when all abnormalities are regarded as fracture signs. 

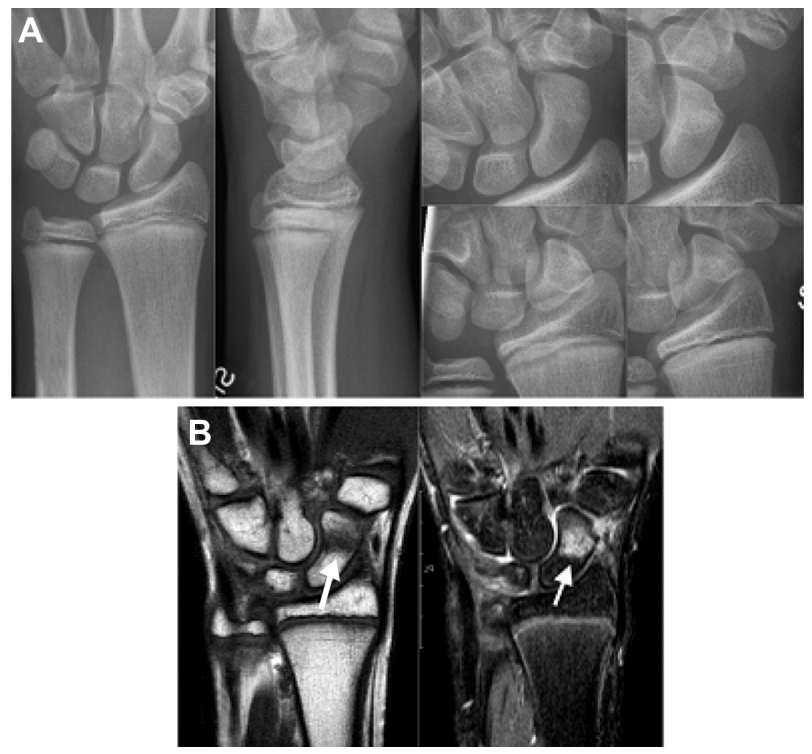

Figure 7 A 14-year-old boy with wrist trauma.

Notes: (A) Radiography including four scaphoid views was entirely unremarkable. (B) MRI done 4 days later with coronal TI-weighted and STIR sequences displayed bone marrow edema across the scaphoid waist, consistent with a non-displaced trabecular bone fracture (arrows).

Abbreviations: MRI, magnetic resonance imaging; STIR, short tau inversion recovery.

A cost-effective scaphoid fracture MRI protocol may consist of a coronal short tau inversion-recovery (STIR) sequence or a similar fluid-sensitive sequence, combined with a turbo spin-echo T1-weighted coronal sequence. Fracture detection is based on high signal on the STIR sequence consistent with bone bruise or hemorrhage combined with a linear low-signal intensity line on the T1-weighted sequence consistent with a fracture line. At the author's institution, patients with or without a cast are examined in a $1.5 \mathrm{~T}$ scanner using a small surface coil with coronal STIR and T1-weighted sequences. The scan parameters are given in Table 1. Patients without plaster are mostly examined in a $3 \mathrm{~T}$ scanner with a dedicated wrist coil, providing thinner slices and generally higher image quality (Table 1). The technique has, however,

Table I An overview of suggested scan parameters for scaphoid trauma imaging at $1.5 \mathrm{~T}$ and $3 \mathrm{~T}$

\begin{tabular}{lllllll}
\hline $\begin{array}{l}\text { Field } \\
\text { strength }\end{array}$ & Type & TR & TE & Matrix & Thickness & Scan time \\
\hline I.5 T & T2 STIR & 2,500 & 70 & $208 \times 163$ & $3 \mathrm{~mm}$ & 4.02 minutes \\
& TI SE & 244 & 16 & $200 \times 200$ & $2.5 \mathrm{~mm}$ & 4.21 minutes \\
$3 \mathrm{~T}$ & T2 STIR & 2,096 & 60 & $240 \times 131$ & $2 \mathrm{~mm}$ & 4.53 minutes \\
& TI TSE & 650 & 23 & $272 \times 216$ & $2 \mathrm{~mm}$ & 2.26 minutes \\
\hline
\end{tabular}

Notes: Both protocols are rapid and dedicated trauma protocols to confirm or rule out scaphoid, or other wrist or carpal bone fractures. For a more comprehensive evaluation of a detected injury additional sequences may be needed.

Abbreviations: TR, repetition time; TE, echo time; STIR, short tau inversionrecovery; SE, spin echo; TSE, turbo spin echo.

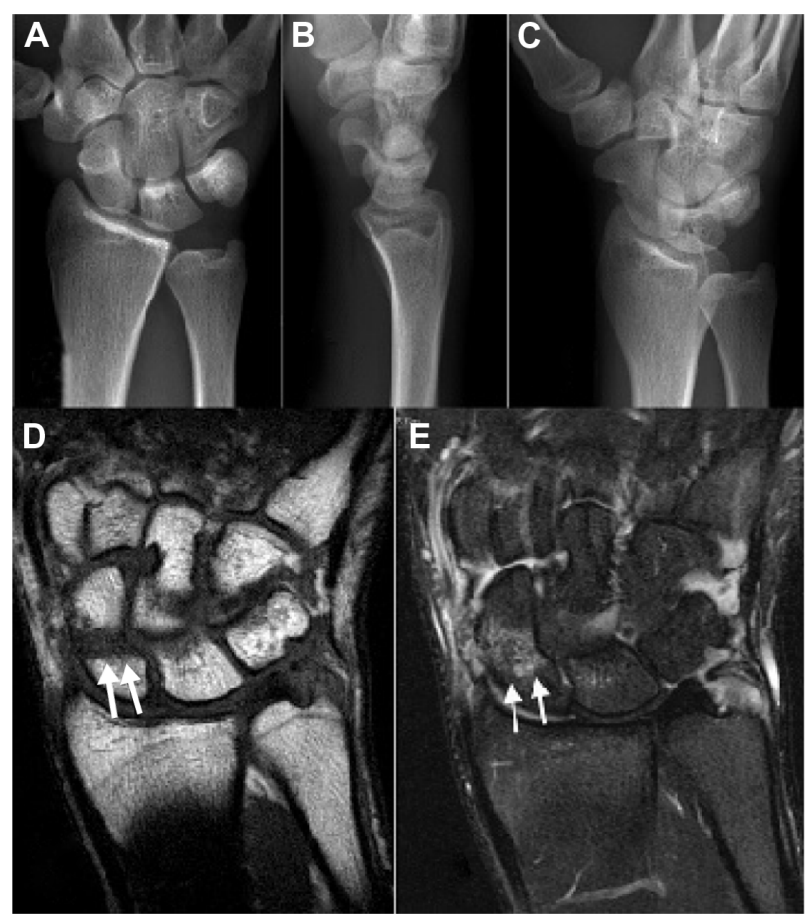

Figure 8 A 17-year-old boy with wrist trauma.

Notes: (A-C) Radiography including a scaphoid view was unremarkable. (D-E) MRI the same day with coronal TI-weighted and fat-saturated T2-weighted sequences displayed bone marrow edema across the scaphoid waist, consistent with a nondisplaced trabecular bone fracture (arrows).

Abbreviation: MRI, magnetic resonance imaging.

difficulties in differentiating between a non-displaced cortical fracture with an inherent potential for displacement, and a purely trabecular fracture seen as a bone bruise (Figure 8). Examination in a plaster or plastic cast is possible, but results in decreased image quality as dedicated wrist coils cannot be used. The advantage of using MRI is that not only will other skeletal injuries also be revealed, similar to bone scanning, but soft tissue injuries will be shown as well. In children, there may be residual high signal in the growing skeleton up to about age 16 years. This should not be confused with bone bruise after trauma. ${ }^{95}$

MRI after normal initial radiography changed patient management in $92 \%$ of 195 patients from normally repeat radiography after 2 weeks to either immediate discharge or clinical review. ${ }^{65}$ In a retrospective analysis on 214 limited scaphoid MRI examinations, no significant fractures in the images were missed. However, several patients with normal MRI had persistent symptoms. ${ }^{96} \mathrm{~A}$ recent study using an examination protocol similar to the one described above ${ }^{97}$ reported a small cost benefit for early MRI over conventional management with repeat radiography, but it also suggested a higher societal gain due to less unnecessary immobilization. Another recent study, adding limited MRI after normal follow-up radiographs ${ }^{98}$ also 
showed similar costs to the standard protocol but reduced societal costs, as have other studies. ${ }^{31,99}$

When using a whole-body scanner some patients cannot be examined due to absolute or relative contraindications, such as a pacemaker, certain metallic implants, or claustrophobia. This problem can be circumvented by using a dedicated extremity MRI unit.

\section{Extremity MRI}

Extremity MRI scanners have been available for several years, ${ }^{66}$ with field strengths ranging from low-field at about $0.2 \mathrm{~T}$ to high-field scanners at $1.0 \mathrm{~T}$ and even $1.5 \mathrm{~T}$. The scanners usually include a narrow and comparatively short tunnel, and allow for imaging of the hand and wrist, elbow, foot and ankle, and knee. The units are light-weight and can be accommodated on a small surface area of about $20 \mathrm{~m}^{2}$, including the operator console and computer equipment. Dedicated extremity scanners have advantages over wholebody scanners in ease of patient positioning, patient comfort, and in terms of contraindications. However, low-field scanners may have longer acquisition times and poorer image quality than high-field scanners. For example, in a study on erosions in rheumatoid arthritis, MRI with a low-field dedicated extremity MRI scanner performed better than radiography, but worse than a whole-body high-field MRI scanner. ${ }^{100}$ In a study on suspected occult scaphoid fractures, however, patient management was altered in $92 \%$ of 195 patients when using a $0.2 \mathrm{~T}$ scanner. ${ }^{65}$ In that study, $19 \%$ of patients had a scaphoid fracture.

\section{Which modality is best, and when?}

There is no international consensus on which imaging modality is best or most cost-efficient for detecting occult scaphoid fractures. In an international survey of hospital practice in imaging scaphoid trauma in 2006, 200 hospitals worldwide were queried on their use of imaging protocols and modalities in suspected scaphoid fractures. ${ }^{101}$ All had access to MRI, bone scanning, and CT. Of the 105 replies, only 23 reported having fixed imaging protocols. In $72 \%$, repeat radiography was performed before continuing with another imaging modality. The choice and timing of further imaging was highly variable, with imaging performed either on the same day as initial radiography or up to more than 2 weeks later. The modality of choice varied, with MRI in preferred $29.5 \%$ of hospitals, CT in $18.1 \%$, and scintigraphy in $13.3 \%$. Combined protocols included CT or MRI in $9.5 \%$, scintigraphy or $\mathrm{CT}$ in $5.7 \%$, and scintigraphy or MRI in $5.7 \%$. Clinical examination and radiography alone were
Table 2 A summary of prices for various scaphoid examinations at the author's institution in 2013 , converted to US dollars

\begin{tabular}{ll}
\hline Examination & Price (USD) \\
\hline Radiography, wrist & $\$ 58$ \\
Radiography, scaphoid views & $\$ 58$ \\
Tomosynthesis, wrist and scaphoid & $\$ 115$ \\
CT, wrist & $\$ 201$ \\
MRI, wrist & $\$ 432$ \\
MRI, scaphoid trauma protocol & $\$ 230$ \\
Bone scintigraphy, scaphoid & $\$ 335$ \\
\hline
\end{tabular}

Abbreviations: USD, United States dollars; CT, computed tomography; MRI, magnetic resonance imaging.

used in $5.7 \%$ of the hospitals. A similar survey was carried out in the United Kingdom directed at members of the British Orthopaedic Association. ${ }^{102}$ Only 16\% of 832 responded, of which only $16 \%$ had knowledge of a local imaging protocol for suspected scaphoid fractures. Clinical and radiographic follow-up was used by $94 \%$. In the $16 \%$ with awareness of a local imaging protocol, MRI was preferred in $58 \%$, CT in $26 \%$, and bone scanning in $16 \%$. Prices of the different examinations, which may certainly play a role in the selection of a modality, varies worldwide, for many reasons. As an example, prices at the author's institution are provided (Table 2), showing the highest cost for bone scintigraphy and full wrist MRI, with scaphoid trauma MRI and wrist CT in the medium range, and radiography and tomosynthesis being least expensive. A recent study from the United Kingdom ${ }^{97}$ reported a slightly lower cost for radiography and a similar cost for scaphoid trauma MRI. Pros and cons for the different modalities have been summarized in Table 3 .

In an analysis by Jenkins et al, ${ }^{64}$ the best and worst performance for MRI, CT, bone scintigraphy, and ultrasound from the available literature was analyzed, including calculations of sensitivity and specificity, and modelled on a patient population of 200 consecutive cases with suspected occult scaphoid fractures (Table 4). In reviews comparing the performance of CT, MRI, and bone scanning, all modalities show high sensitivity and specificity. ${ }^{93,103}$ MRI has been

Table 3 Summary of imaging characteristics for radiologic modalities applied in scaphoid fracture imaging

\begin{tabular}{llll}
\hline Modality & Availability & Cost & Radiation \\
\hline Radiography & Good & Low & Minimal \\
Tomosynthesis & Limited & Low & Minimal \\
Ultrasound & Moderate - good & Low-moderate & None \\
CT & Moderate - good & Moderate & Minimal \\
MRI & Moderate & High & None \\
Bone scintigraphy & Moderate & High & High \\
\hline
\end{tabular}

Abbreviations: $\mathrm{CT}$, computed tomography; MRI, magnetic resonance imaging. 
Table 4 Diagnostic characteristics of scaphoid imaging techniques as described by Jenkins et $\mathrm{al}^{64}$, reporting the best and worst figures for sensitivity and specificity from the literature

\begin{tabular}{|c|c|c|c|}
\hline Investigation & Reference standard & Sensitivity & Specificity \\
\hline \multicolumn{4}{|l|}{$\overline{C T}$} \\
\hline Best ${ }^{105}$ & $\begin{array}{l}\text { Bone scintigraphy } \\
\text { and delayed CT }\end{array}$ & 1 & 1 \\
\hline Worst $^{16}$ & $\begin{array}{l}\text { Delayed radiography } \\
\text { and normal controls }\end{array}$ & 0.97 & 0.85 \\
\hline \multicolumn{4}{|l|}{ Ultrasound } \\
\hline Best $^{89}$ & Delayed radiography & I & 0.98 \\
\hline Worst ${ }^{106}$ & Delayed radiography & 0.5 & 0.91 \\
\hline \multicolumn{4}{|c|}{ Bone scintigraphy } \\
\hline Best $^{87}$ & Delayed radiography & 1 & 0.98 \\
\hline Worst ${ }^{87}$ & Delayed radiography & 0.77 & 0.88 \\
\hline \multicolumn{4}{|l|}{ MRI } \\
\hline Best $^{107}$ & Delayed radiography & I & I \\
\hline Worst ${ }^{63}$ & Delayed radiography & 0.98 & I \\
\hline
\end{tabular}

Notes: Reprinted (in part) from Injury, Jenkins PJ, Slade K, Huntley JS, Robinson CM, A comparative analysis of the accuracy, diagnostic uncertainty and cost of imaging modalities in suspected scaphoid fractures, 39(7): 768-774, Copyright 2008 with permission from Elsevier. ${ }^{64}$

Abbreviations: $\mathrm{CT}$, computed tomography; MRI, magnetic resonance imaging.

shown to be cost-effective in detecting occult scaphoid fractures, ${ }^{31,104}$ and so too, probably, are CT and bone scanning.

\section{Summary}

There is no clear consensus in the choice of final imaging in suspected occult fracture, with a large variation in imaging and clinical protocols. Cost and access are not the sole factors determining the choice of modality, but also physicians' own opinions. Most guidelines are based on older research, and there is still perhaps a lack of research to determine which modality has the highest clinical utility for a given situation. It would appear highly unnecessary to treat patients without a scaphoid or other significant fracture in a cast for 2 or even 6 weeks just to be on the safe side, or to subject patients without a scaphoid or other significant fracture to further clinical follow-up and imaging. Potentially, it may be unnecessary to treat purely trabecular scaphoid fractures without cortical disruption with casting, other than for the purpose of pain relief. Several questions remain to be answered: Which occult scaphoid fractures are vital to detect - all fractures, or only those with a potential for later displacement? Is it clinically important to detect fractures in other carpal bones than the scaphoid? Is it important to detect purely trabecular scaphoid fractures?

The various imaging modalities have various inherent problems. Radiography fails to detect a number of occult fractures. CT fails to detect purely trabecular fractures. There may be false positive results with MRI, and MRI may lead to overdiagnosis and overtreatment, which is also the case with bone scanning. Ultrasound is operator-dependent and time-consuming. On the positive side, there is a rapid advance in imaging technique and quality for MRI as well as for CT. Extremity scanners with high image quality are available for both MRI and CT. Bone scanning may see a new dawn with SPECT-CT.

In conclusion, evaluation of a suspected scaphoid fracture should start with wrist radiography supplemented by special scaphoid views. Follow-up investigation when a radiographically occult scaphoid fracture is suspected should be done with another imaging modality; the most commonly preferred modality for follow-up at present is MRI. There are, however, other options such as CT and bone scanning which provide similar results. Modern comparative studies are needed, to improve scaphoid fracture detection, and to help determine which patients need which treatment.

\section{Disclosure}

The author reports no conflicts of interest in this work.

\section{References}

1. Meermans G, Verstreken F. Influence of screw design, sex, and approach in scaphoid fracture fixation. Clin Orthop. 2012;470(6):1673-1681.

2. Cooney WP, Linscheid RL, Dobyns JH. Scaphoid fractures. Problems associated with nonunion and avascular necrosis. Orthop Clin North Am. 1984;15(2):381-391.

3. Taleisnik J, Kelly PJ. Extraosseous and intraosseous blood supply of the scaphoid bone. J Bone Joint Surg Am. 1966;48(6):1125-1137.

4. Gelberman RH, Gross MS. The vascularity of the wrist. Identification of arterial patterns at risk. Clin Orthop. 1986;202:40-49.

5. Gelberman RH, Menon J. The vascularity of the scaphoid bone. J Hand Surg Am. 1980;5(5):508-513.

6. Panagis JS, Gelberman RH, Taleisnik J, Baumgaertner M. The arterial anatomy of the human carpus. Part II: the intraosseous vascularity. J Hand Surg Am. 1983;8(4):375-382.

7. Rhemrev SJ, Ootes D, Beeres FJ, Meylaerts SA, Schipper IB. Current methods of diagnosis and treatment of scaphoid fractures. Int J Emerg Med. 2011;4:4

8. Cockshott WP. Distal avulsion fractures of the scaphoid. Br J Radiol. 1980;53(635):1037-1040

9. RUSSE O. Fracture of the carpal navicular. Diagnosis, non-operative treatment, and operative treatment. J Bone Joint Surg Am. 1960;42-A: 759-768.

10. Herbert TJ, Fisher WE. Management of the fractured scaphoid using a new bone screw. J Bone Joint Surg Br. 1984;66(1):114-123.

11. Burge P. Closed cast treatment of scaphoid fractures. Hand Clin. 2001;17(4):541-552.

12. Filan SL, Herbert TJ. Herbert screw fixation of scaphoid fractures. J Bone Joint Surg Br. 1996;78(4):519-529.

13. Compson JP. The anatomy of acute scaphoid fractures: a threedimensional analysis of patterns. J Bone Joint Surg Br. 1998;80(2): 218-224.

14. Dias JJ, Wildin CJ, Bhowal B, Thompson JR. Should acute scaphoid fractures be fixed? A randomized controlled trial. J Bone Joint Surg Am. 2005;87(10):2160-2168. 
15. Lozano-Calderón S, Blazar P, Zurakowski D, Lee SG, Ring D. Diagnosis of scaphoid fracture displacement with radiography and computed tomography. J Bone Joint Surg Am. 2006;88(12):2695-2703.

16. Adey L, Souer JS, Lozano-Calderon S, Palmer W, Lee SG, Ring D. Computed tomography of suspected scaphoid fractures. J Hand Surg Am. 2007;32(1):61-66.

17. Duckworth AD, Jenkins PJ, Aitken SA, Clement ND, Court-Brown CM, McQueen MM. Scaphoid fracture epidemiology. J Trauma Acute Care Surg. 2012;72(2):E41-E45.

18. Mody BS, Belliappa PP, Dias JJ, Barton NJ. Nonunion of fractures of the scaphoid tuberosity. J Bone Joint Surg Br. 1993;75(3):423-425.

19. Yin ZG, Zhang JB, Kan SL, Wang XG. Treatment of acute scaphoid fractures: systematic review and meta-analysis. Clin Orthop. 2007;460: $142-151$

20. Rhemrev SJ, van Leerdam RH, Ootes D, Beeres FJ, Meylaerts SA. Non-operative treatment of non-displaced scaphoid fractures may be preferred. Injury. 2009;40(6):638-641.

21. Bond CD, Shin AY, McBride MT, Dao KD. Percutaneous screw fixation or cast immobilization for nondisplaced scaphoid fractures. J Bone Joint Surg Am. 2001;83-A(4):483-488.

22. Vinnars B, Pietreanu M, Bodestedt A, Ekenstam F, Gerdin B. Nonoperative compared with operative treatment of acute scaphoid fractures. A randomized clinical trial. J Bone Joint Surg Am. 2008;90(6):1176-1185.

23. Geissler WB, Adams JE, Bindra RR, Lanzinger WD, Slutsky DJ. Scaphoid fractures: what's hot, what's not. Instr Course Lect. 2012;61:71-84.

24. Fowler JR, Ilyas AM. Headless compression screw fixation of scaphoid fractures. Hand Clin. 2010;26(3):351-361, vi.

25. Szabo RM, Manske D. Displaced fractures of the scaphoid. Clin Orthop. 1988;230:30-38

26. Rettig ME, Raskin KB. Retrograde compression screw fixation of acute proximal pole scaphoid fractures. J Hand Surg Am. 1999;24(6): 1206-1210.

27. Buijze GA, Doornberg JN, Ham JS, Ring D, Bhandari M, Poolman RW. Surgical compared with conservative treatment for acute nondisplaced or minimally displaced scaphoid fractures: a systematic review and meta-analysis of randomized controlled trials. J Bone Joint Surg Am. 2010;92(6):1534-1544.

28. Merrell GA, Wolfe SW, Slade JF 3rd. Treatment of scaphoid nonunions: quantitative meta-analysis of the literature. J Hand Surg Am 2002;27(4):685-691.

29. Mallee W, Doornberg JN, Ring D, Niek van Dijk C, Maas M, Goslings JC. Comparison of CT and MRI for diagnosis of suspected scaphoid fractures. J Bone Joint Surg Am. 2011;93(1):20-28.

30. Rhemrev SJ, de Zwart AD, Kingma LM, et al. Early computed tomography compared with bone scintigraphy in suspected scaphoid fractures. Clin Nucl Med. 2010;35(12):931-934.

31. Dorsay TA, Major NM, Helms CA. Cost-effectiveness of immediate MR imaging versus traditional follow-up for revealing radiographically occult scaphoid fractures. AJR Am J Roentgenol. 2001;177(6): 1257-1263.

32. Larson B, Light TR, Ogden JA. Fracture and ischemic necrosis of the immature scaphoid. J Hand Surg Am. 1987;12(1):122-127.

33. Hove LM. Epidemiology of scaphoid fractures in Bergen, Norway. Scand J Plast Reconstr Surg Hand Surg. 1999;33(4):423-426.

34. Dunn AW. Fractures and dislocations of the carpus. Surg Clin North Am. 1972;52(6):1513-1538.

35. van der Molen AB, Groothoff JW, Visser GJ, Robinson PH, Eisma WH. Time off work due to scaphoid fractures and other carpal injuries in The Netherlands in the period 1990 to 1993. J Hand Surg Am. 1999;24(2): 193-198.

36. van Onselen EB, Karim RB, Hage JJ, Ritt MJ. Prevalence and distribution of hand fractures. J Hand Surg Am. 2003;28(5):491-495.

37. Hey HW, Chong AK, Murphy D. Prevalence of carpal fracture in Singapore. J Hand Surg Am. 2011;36(2):278-283.

38. Brøndum V, Larsen CF, Skov O. Fracture of the carpal scaphoid: frequency and distribution in a well-defined population. Eur J Radiol. 1992;15(2):118-122.
39. Hove LM. Simultaneous scaphoid and distal radial fractures. J Hand Surg Br. 1994;19(3):384-388.

40. Oskam J, De Graaf JS, Klasen HJ. Fractures of the distal radius and scaphoid. J Hand Surg Br. 1996;21(6):772-774.

41. Smith JT, Keeve JP, Bertin KC, Mann RJ. Simultaneous fractures of the distal radius and scaphoid. J Trauma. 1988;28(5):676-679.

42. Albert MC, Barre PS. A scaphoid fracture associated with a displaced distal radial fracture in a child. Clin Orthop. 1989;240:232-235.

43. Lahoti O, Wong J, Regan B, Fogarty EE, Dowling FE. Fracture of the scaphoid associated with volar displacement of a lower radial epiphyseal fracture. Case report. Scand J Plast Reconstr Surg Hand Surg. 1993;27(2):155-156.

44. Richards RR, Ghose T, McBroom RJ. Ipsilateral fractures of the distal radius and scaphoid treated by Herbert screw and external skeletal fixation. A report of two cases. Clin Orthop. 1992;282:219-221.

45. Stother IG. A report of 3 cases of simultaneous Colles' and scaphoid fractures. Injury. 1976;7(3):185-188.

46. Pillai A, Jain M. Management of clinical fractures of the scaphoid: results of an audit and literature review. Eur J Emerg Med. 2005;12(2): 47-51.

47. Rhemrev SJ, Beeres FJP, van Leerdam RH, Hogervorst M, Ring D. Clinical prediction rule for suspected scaphoid fractures: a prospective cohort study. Injury. 2010;41(1):1026-1030.

48. Unay K, Gokcen B, Ozkan K, Poyanli O, Eceviz E. Examination tests predictive of bone injury in patients with clinically suspected occult scaphoid fracture. Injury. 2009;40(12):1265-1268.

49. Hackney LA, Dodds SD. Assessment of scaphoid fracture healing. Curr Rev Musculoskelet Med. 2011;4(1):16-22.

50. Phillips TG, Reibach AM, Slomiany WP. Diagnosis and management of scaphoid fractures. Am Fam Physician. 2004;70(5):879-884.

51. Schubert HE. Scaphoid fracture. Review of diagnostic tests and treatment. Can Fam Physician. 2000;46:1825-1832.

52. Chen SC. The scaphoid compression test. J Hand Surg Am. 1989;14(3): 323-325.

53. Parvizi J, Wayman J, Kelly P, Moran CG. Combining the clinical signs improves diagnosis of scaphoid fractures. A prospective study with follow-up. J Hand Surg Br. 1998;23(3):324-327.

54. Grover R. Clinical assessment of scaphoid injuries and the detection of fractures. J Hand Surg Am. 1996;21(3):341-343.

55. Buijze GA, Mallee WH, Beeres FJP, Hanson TE, Johnson WO, Ring D. Diagnostic performance tests for suspected scaphoid fractures differ with conventional and latent class analysis. Clin Orthop. 2011;469(12): 3400-3407.

56. Yin ZG, Zhang JB, Kan SL, Wang XG. Diagnostic accuracy of imaging modalities for suspected scaphoid fractures: meta-analysis combined with latent class analysis. J Bone Joint Surg Br. 2012;94(8): 1077-1085.

57. Low G, Raby N. Can follow-up radiography for acute scaphoid fracture still be considered a valid investigation? Clin Radiol. 2005;60(10): $1106-1110$

58. Geijer M, Börjesson AM, Göthlin JH. Clinical utility of tomosynthesis in suspected scaphoid fracture. A pilot study. Skeletal Radiol. 2011;40(7):863-867.

59. Törnvall AH, af Ekenstam F, Hagert CG, Irstam L. Radiologic examination and measurement of the wrist and distal radio-ulnar joint. New aspects. Acta Radiol Diagn (Stockh). 1986;27(5):581-588.

60. Jedlinski A, Kauer JM, Jonsson K. X-ray evaluation of the true neutral position of the wrist: the groove for extensor carpi ulnaris as a landmark. J Hand Surg Am. 1995;20(3):511-512.

61. Dias JJ, Thompson J, Barton NJ, Gregg PJ. Suspected scaphoid fractures The value of radiographs. J Bone Joint Surg Br. 1990;72(1):98-101.

62. Tiel-van Buul MM, van Beek EJ, Broekhuizen AH, Nooitgedacht EA, Davids PH, Bakker AJ. Diagnosing scaphoid fractures: radiographs cannot be used as a gold standard! Injury. 1992;23(2):77-79.

63. Hunter JC, Escobedo EM, Wilson AJ, Hanel DP, Zink-Brody GC, Mann FA. MR imaging of clinically suspected scaphoid fractures. AJR Am J Roentgenol. 1997;168(5):1287-1293. 
64. Jenkins PJ, Slade K, Huntley JS, Robinson CM. A comparative analysis of the accuracy, diagnostic uncertainty and cost of imaging modalities in suspected scaphoid fractures. Injury. 2008;39(7):768-774.

65. Brydie A, Raby N. Early MRI in the management of clinical scaphoid fracture. Br J Radiol. 2003;76(905):296-300.

66. Bretlau T, Christensen OM, Edstrom P, Thomsen HS, Lausten GS. Diagnosis of scaphoid fracture and dedicated extremity MRI. Acta Orthop Scand. 1999;70(5):504-508.

67. Dobbins JT, Godfrey DJ. Digital x-ray tomosynthesis: current state of the art and clinical potential. Phys Med Biol. 2003;48(19): R65-R106.

68. Park JM, Franken EA Jr, Garg M, Fajardo LL, Niklason LT. Breast tomosynthesis: present considerations and future applications. Radiographics. 2007;27 Supp1 1:S231-S240.

69. Ottenin MA, Jacquot A, Grospretre O, et al. Evaluation of the diagnostic performance of tomosynthesis in fractures of the wrist. AJR Am J Roentgenol. 2012;198(1):180-186.

70. Geijer M, El-Khoury GY. MDCT in the evaluation of skeletal trauma: principles, protocols, and clinical applications. Emerg Radiol. 2006;13(1):7-18.

71. Buckwalter KA, Rydberg J, Kopecky KK, Crow K, Yang EL. Musculoskeletal imaging with multislice CT. AJR Am J Roentgenol. 2001;176(4):979-986.

72. Sanders WE. Evaluation of the humpback scaphoid by computed tomography in the longitudinal axial plane of the scaphoid. J Hand Surg Am. 1988;13(2):182-187.

73. Lozano-Calderón SA, Doornberg J, Ring D. Fractures of the dorsal articular margin of the distal part of the radius with dorsal radiocarpal subluxation. J Bone Joint Surg Am. 2006;88(7):1486-1493.

74. Ty JM, Lozano-Calderon S, Ring D. Computed tomography for triage of suspected scaphoid fractures. Hand (NY). 2008;3(2):155-158.

75. Biswas D, Bible JE, Bohan M, Simpson AK, Whang PG, Grauer JN. Radiation exposure from musculoskeletal computerized tomographic scans. J Bone Joint Surg Am. 2009;91(8):1882-1889.

76. Geijer M, Collin D, Dunker D, Göthlin JH. Bone bruise, lipohemarthrosis, and joint effusion in CT of non-displaced hip fracture. Acta Radiol. 2012;53(2):197-202.

77. Pache G, Krauss B, Strohm P, et al. Dual-energy CT virtual noncalcium technique: detecting posttraumatic bone marrow lesions - feasibility study. Radiology. 2010;256(2):617-624.

78. Stevenson JD, Morley D, Srivastava S, Willard C, Bhoora IG. Early CT for suspected occult scaphoid fractures. J Hand Surg Eur Vol. 2012;37(5): $447-451$.

79. Memarsadeghi M, Breitenseher MJ, Schaefer-Prokop C, et al. Occult scaphoid fractures: comparison of multidetector CT and MR imaging - initial experience. Radiology. 2006;240(1):169-176.

80. Zbijewski W, De Jean P, Prakash P, et al. A dedicated cone-beam CT system for musculoskeletal extremities imaging: design, optimization, and initial performance characterization. Med Phys. 2011;38(8):4700-4713.

81. Koskinen SK, Haapamäki VV, Salo J, et al. CT arthrography of the wrist using a novel, mobile, dedicated extremity cone-beam CT (CBCT). Skeletal Radiol. 2013;42(5):649-657.

82. Citak M, O’Loughlin PF, Kendoff D, et al. Navigated scaphoid screw placement using customized scaphoid splint: an anatomical study. Arch Orthop Trauma Surg. 2010;130(7):889-895.

83. Smith EJ, Al-Sanawi HA, Gammon B, St John PJ, Pichora DR, Ellis RE. Volume slicing of cone-beam computed tomography images for navigation of percutaneous scaphoid fixation. Int J Comput Assist Radiol Surg. 2012;7(3):433-444.

84. McDougall IR, Rieser RP. Scintigraphic techniques in musculoskeletal trauma. Radiol Clin North Am. 1989;27(5):1003-1011.
85. Horger M, Bares R. The role of single-photon emission computed tomography/computed tomography in benign and malignant bone disease. Semin Nucl Med. 2006;36(4):286-294.

86. Brismar J. Skeletal scintigraphy of the wrist in suggested scaphoid fracture. Acta Radiol. 1988;29(1):101-107.

87. Tiel-van Buul MM, van Beek EJ, Borm JJ, Gubler FM, Broekhuizen AH, van Royen EA. The value of radiographs and bone scintigraphy in suspected scaphoid fracture. J Hand Surg Br. 1993;18(3):403-406.

88. Murphy DG, Eisenhauer MA, Powe J, Pavlofsky W. Can a day 4 bone scan accurately determine the presence or absence of scaphoid fracture? Ann Emerg Med. 1995;26(4):434- 438.

89. Fowler C, Sullivan B, Williams LA, McCarthy G, Savage R, Palmer A. A comparison of bone scintigraphy and MRI in the early diagnosis of the occult scaphoid waist fracture. Skeletal Radiol. 1998;27(12): 683-687.

90. Groves AM, Cheow H, Balan K, Courtney H, Bearcroft P, Dixon A. 16-MDCT in the detection of occult wrist fractures: a comparison with skeletal scintigraphy. AJR Am J Roentgenol. 2005;184(5): 1470-1474.

91. Henriksen OM, Lonsdale MN, Jensen TD, et al. Two-dimensional fusion imaging of planar bone scintigraphy and radiographs in patients with clinical scaphoid fracture: an imaging study. Acta Radiol. 2009;50(1):71-77.

92. Hauger O, Bonnefoy O, Moinard M, Bersani D, Diard F. Occult fractures of the waist of the scaphoid: early diagnosis by high-spatial-resolution sonography. AJR Am J Roentgenol. 2002;178(5):1239-1245.

93. Ring D, Lozano-Calderón S. Imaging for suspected scaphoid fracture. J Hand Surg Am. 2008;33(6):954-957.

94. Karantanas A, Dailiana Z, Malizos K. The role of MR imaging in scaphoid disorders. Eur Radiol. 2007;17(11):2860-2871.

95. Shabshin N, Schweitzer ME. Age dependent T2 changes of bone marrow in pediatric wrist MRI. Skeletal Radiol. 2009;38(12):1163-1168.

96. Bowles F, Keeton H, Adlington H, Cumberbatch GL, Markham D. Morbidity in adults with a normal limited scaphoid MRI: a retrospective cohort study and follow-up questionnaire. Emerg Med J. 2013;30(3):208-210.

97. Patel NK, Davies N, Mirza Z, Watson M. Cost and clinical effectiveness of MRI in occult scaphoid fractures: a randomised controlled trial. Emerg Med J. 2013;30(3):202-207.

98. Moreno Ramos MD, Martinez Hervas M, Sanz Rupp P, Ramos Medrano J. Analysis of the management of occult fractures of the scaphoid through early magnetic resonance imaging. Radiologia. 2013;55(3):247-252. Spanish.

99. Hansen TB, Petersen RB, Barckman J, Uhre P, Larsen K. Costeffectiveness of MRI in managing suspected scaphoid fractures. J Hand Surg Eur Vol. 2009;34(5):627-630.

100. Freeston JE, Conaghan PG, Dass S, et al. Does extremity-MRI improve erosion detection in severely damaged joints? A study of long-standing rheumatoid arthritis using three imaging modalities. Ann Rheum Dis. 2007;66(11):1538-1540.

101. Groves AM, Kayani I, Syed R, et al. An international survey of hospital practice in the imaging of acute scaphoid trauma. AJR Am J Roentgenol. 2006;187(6):1453-1456.

102. Brookes-Fazakerley SD, Kumar AJ, Oakley J. Survey of the initial management and imaging protocols for occult scaphoid fractures in UK hospitals. Skeletal Radiol. 2009;38(11):1045-1048.

103. Yin ZG, Zhang JB, Kan SL, Wang XG. Diagnosing suspected scaphoid fractures: a systematic review and meta-analysis. Clin Orthop. 2010;468(3):723-734

104. Brooks S, Cicuttini FM, Lim S, Taylor D, Stuckey SL, Wluka AE. Cost effectiveness of adding magnetic resonance imaging to the usual management of suspected scaphoid fractures. Br J Sports Med. 2005;39(2):75-79. 
105. Breederveld RS, Tuinebreijer WE. Investigation of computed tomographic scan concurrent criterion validity in doubtful scaphoid fracture of the wrist. J Trauma. 2004;57(4):851-854.

106. Munk B, Bolvig L, Krøner K, Christiansen T, Borris L, Boe S. Ultrasound for diagnosis of scaphoid fractures. J Hand Surg Br. 2000;25(4):369-371.
107. Gaebler C, Kukla C, Breitenseher M, Trattnig S, Mittlboeck M, Vécsei V. Magnetic resonance imaging of occult scaphoid fractures. J Trauma. 1996;41(1):73-76.

Reports in Medical Imaging

\section{Publish your work in this journal}

Reports in Medical Imaging is an international, peer-reviewed, open access journal publishing original research, reports, reviews and commentaries on all areas of medical imaging. The manuscript management system is completely online and includes a very quick and fair peer-review system, which is all easy to use.

\section{Dovepress}

Visit http://www.dovepress.com/testimonials.php to read real quotes from published authors. 